\title{
Blended Learning as Instructional Model in Vocational Education: Literature Review
}

\author{
Krismadinata ${ }^{1}$, Unung Verawardina ${ }^{1,2, *}$, Nizwardi Jalinus ${ }^{1}$, Fahmi Rizal $^{1}$, Sukardi ${ }^{1}$, Putu Sudira ${ }^{3}$, \\ Dochi Ramadhani ${ }^{2}$, Arina Luthfini Lubis ${ }^{4}$, John Friadi ${ }^{5}$, Ari Syaiful Rahman Arifin ${ }^{1}$, \\ Dony Novaliendry ${ }^{1,6}$ \\ ${ }^{1}$ Faculty of Engineering, Universitas Negeri Padang, Padang, Indonesia \\ ${ }^{2}$ Information Technology and Computer Education, IKIP PGRI Pontianak, Pontianak, Indonesia \\ ${ }^{3}$ Technology and Vocational Education, Universitas Negeri Yogyakarta, Yogyakarta, Indonesia \\ ${ }^{4}$ Faculty of Engineering, Universitas Ibnu Sina Batam, Batam, Indonesia \\ ${ }^{5}$ Department of Information System, Faculty of Engineering, Universitas Batam, Batam, Indonesia \\ ${ }^{6}$ Department of Electronic, National Kaohsiung University of Science and Technology, Kaohsiung, Taiwan
}

Received July 6, 2020; Revised August 28, 2020; Accepted October 19, 2020

\section{Cite This Paper in the following Citation Styles}

(a): [1] Krismadinata, Unung Verawardina, Nizwardi Jalinus, Fahmi Rizal, Sukardi, Putu Sudira, Dochi Ramadhani, Arina Luthfini Lubis, John Friadi, Ari Syaiful Rahman Arifin, Dony Novaliendry, "Blended Learning as Instructional Model in Vocational Education: Literature Review," Universal Journal of Educational Research, Vol. 8, No. 11B, pp. 5801 - 5815, 2020. DOI: 10.13189/ujer.2020.082214.

(b): Krismadinata, Unung Verawardina, Nizwardi Jalinus, Fahmi Rizal, Sukardi, Putu Sudira, Dochi Ramadhani, Arina Luthfini Lubis, John Friadi, Ari Syaiful Rahman Arifin, Dony Novaliendry (2020). Blended Learning as Instructional Model in Vocational Education: Literature Review. Universal Journal of Educational Research, 8(11B), 5801 - 5815. DOI: 10.13189/ujer.2020.082214.

Copyright $\bigcirc 2020$ by authors, all rights reserved. Authors agree that this article remains permanently open access under the terms of the Creative Commons Attribution License 4.0 International License

\begin{abstract}
This article aims to examine and provide an explanation of the blended learning model in vocational education. The method used is a meta-analysis method. All data contained in published studies were all used. Sampling suitable for meta-analysis using surveys, laboratory experiments and field studies is based on the results of literature studies from pre-existing research, covering 45 publications, through studies of scientific sources, indexed international journals and relevant books, and also from several findings. Based on the research results, blended learning is a learning process that combines face-to-face learning and online learning. Before implementing blended learning in vocational education, things that need to be considered first for the implementation of blended learning are the normal development of the blended learning model, application of topology, and also knowing the characteristics of the institution. Particularly in the field of vocational, one must be able to adapt to vocational education, infrastructure, technology, learning, pedagogical principles, activity assessment, baiting processes, interactions, resources, activities, infrastructure, culture, management and organization, ethics, etc.
\end{abstract}

Furthermore, it is also necessary to make the blended learning stage more systematic, starting from the stage of creating conditions for success, planning, implementing, and improving with the sub-stages. There were four blended learning models to choose from, namely the rotation model (consisting of rotation model, lab rotation, and flipped individual classroom rotation), flex model, self-blend model, and enriched-virtual model.

Keywords Model Blended Learning, Vocational Education, Online Learning, Face to Face Learning

\section{Introduction}

The development of digital technology creates one innovation in learning models that can occur both online and offline (known as blended learning). Blended learning is a formal education program through the delivery of content and instruction through digital and online media that control elements of students over time, place, and 
speed [1][48][49][50][51][52][53][54][55]. Many universities implement a mixed learning process because it can promote effective learning and successfully integrate online and offline classes for adult students [2]. Teachers who are involved in mixed learning processes in vocational education, tend to adopt different approaches to teaching as well as mixed learning designs [3].

The advantage of blended learning is that students can learn flexibly, freely, and broadly wherever and whenever [4]. Blended models can improve student's performance in vocational education and lead to more effective learning. [5]. Blended learning can reduce the number of class meetings [6]. The blended learning model gives teachers more time in learning activities that involve students in improving their abilities.

The facts that occur in very boring learning activities will make students less enthusiastic about learning and even fall asleep [7] [8].

Learning that combines online and offline classes will be able to build interactions, create projects, be able to access materials in the form of online teaching materials such as percentages, videos, interactive multimedia, and others [4] [47]. The blended learning can also use Learning Management Systems (LMS) because it can distribute subject matter, manage tasks and assignments, and can discuss the subject. Implementing blended learning requires appropriate content through technology such as web 2.0, which can be a suitable choice to improve skills and as a motivation for students in vocational education [9]. Thus, students become more flexible in choosing the time and place of study, for example, in parks, libraries, at home, on trips, in places to hang out like cafes, and others. Even with more teaching materials obtained, the knowledge gained is also broader; and teachers and students can be involved in the teaching and learning process. What needs to be considered in applying a learning model is the teacher's ability to understand the entire learning process according to the expected target [8]. The use of communication and information technology in education is very important in the digital era [10] [11] [12] [13]. The use of technology can increase the competitiveness of human resources in the 21st century [14] [15] [16].

In the learning process, it was found that there were some significant differences in digital literacy skills between the control class and the experimental class, which means that the application of a blended model can improve students' digital literacy skills in vocational education [17]. Blended e-learning does not have a significant effect on student achievement test scores, but it does significantly affect student self-assessment scores in vocational education [18]. The results showed that vocational students and teachers also agreed that there must be a development of critical thinking skills, learning achievement, and learning management in vocational education [19]. Based on these findings, blended learning is very influential in vocational education.
Blended learning can be applied as a supplement and complement or substitute in learning, which includes the content of teaching material in the form of documents, videos, animations, or simulations, and others [20]. Therefore, in its application, blended learning can be used as a supplement, complement, and substitute according to the needs [21][22]. In determining proportions, the characteristics of the courses applied to blended learning must be considered. The learning settings in the blended learning model consist of synchronous and asynchronous.

Direct synchronization is learning that occurs when being in the same location/space and time. At the same time, virtual synchronous is learning in the same space and time but in different locations. Then there is the term asynchronous independent learning that occurs in independent learning situations online. Meanwhile, asynchronous collaborative learning occurs when students collaborate with each other [23].

There are a framework and model of blended learning to carry out the learning process. The blended learning model is divided into several categories consisting of rotation model, lab rotation, flipped classroom, individual rotation, flex model, self-blend model, and enriched virtual model $[24][25][26][27][28]$. Therefore, the instructor can apply the blended models or even develop the model to be used in the teaching and learning process. To design and also implement blended learning, universities must pay attention to the interaction between student design features, and make learning outcomes an indicator of the effectiveness of the blended learning model [29].

Therefore, it needs to have exact steps to plan, develop, and implement a blended learning model using face-to-face and online learning to produce effective learning. This article aimed to examine the development of a blended learning model, to produce precise information and provide an overview of the blended learning model that is ideal for vocational education.

\section{Methodology}

This study uses a meta-analysis method, known as a systematic review, which contains explicit and detailed methods for the identification, selection and assessment of the quality of individual studies and overall evidence but does not incorporate the overall study results.

The meta-analysis uses data contained in published studies. Samples suitable for meta-analysis were collected through surveys, laboratory experiments, as well as field studies based on the results of literature studies from pre-existing research, using 45 publications.

Through the study of scientific sources, such as indexed international journals and relevant books, the findings can also summarize the search and assessment process as shown in Figure 1. 


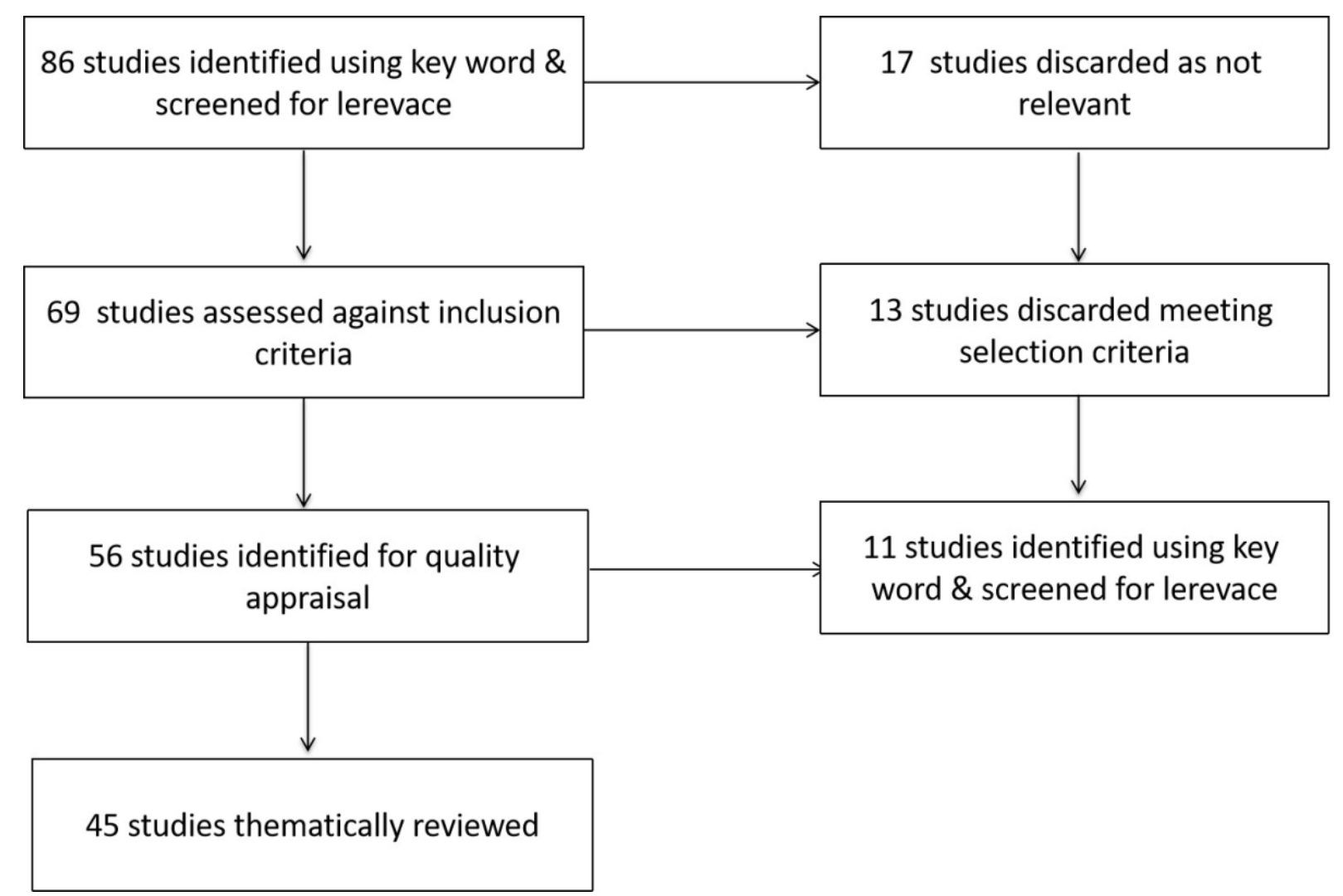

Figure 1. Summary of the search process

Based on Figure 1 about a summary of the search and assessment process, journal sources find studies that can be identified using keywords and are also filtered about blended learning, online, offline, online, blended models, vocational, traditional learning, learning flexibility for a total of 86 lessons. . The 17 lessons carried out were discarded because they were irrelevant, while the other 69 were assessed against the total inclusion criteria. 13 lessons were discarded to meet the selection criteria. Meanwhile, 56 other lessons were identified for quality assessment. A total of 11 studies were identified using keywords and filtered for relevance until finally using 45 thematic studies were reviewed.

\section{Results}

The articles that have been collected must be examined one by one. In the first stage, it must be ascertained whether all are in accordance with the predetermined selection criteria. If the initial search results are extensive, filtering can be done by assessing the abstract of each article. After making sure that it fits the criteria, each study report is assessed for its quality by the researcher.

The explicit criteria for assessing the quality of research articles included the design, implementation and topic analysis aspects of 45 published articles. The results summary of qualitative studies can be seen in table 1 . 
Table 1. Summary of qualitative studies

\begin{tabular}{|c|c|c|c|c|c|c|c|}
\hline No & Author(s) year & Location & $\begin{array}{c}\text { Sample size type and } \\
\text { setting }\end{array}$ & Methodology & Publisher/Source & Key findings & Limitations \\
\hline 1. & $\begin{array}{l}\text { Josephine Adekola, } \\
\text { Vicki H.M. Dale and } \\
\text { Kerr Gardiner. (2017) }\end{array}$ & $\begin{array}{c}\text { University of } \\
\text { Glasgow, Glasgow, } \\
\text { UK }\end{array}$ & $\begin{array}{l}\text { Anggota manajemen } \\
\text { senior dan kepala dinas, } \\
\text { guru, teknolog belajar, } \\
\text { dan anggota Dewan } \\
\text { Perwakilan Siswa } \\
\text { sebanyak } 20 \text { orang } \\
\end{array}$ & Descriptive & $\begin{array}{c}\text { Research in Learning } \\
\text { Technology Vol. 25, } 2017\end{array}$ & $\begin{array}{c}\text { digital education; } \\
\text { technology-enhanced learning; } \\
\text { e-learning; higher education; change }\end{array}$ & $\begin{array}{l}\text { little research sample } \\
\text { No experiment }\end{array}$ \\
\hline 2 & $\begin{array}{c}\text { John Bailey, Carri } \\
\text { Schneider, Tom Vander } \\
\text { Ark. (2015) }\end{array}$ & - & - & - & by Digital Learning Now! & $\begin{array}{l}\text { We are navigating The Digital Shift: } \\
\text { Implementation Strategies For } \\
\text { Blended And Online Learning. } \\
\text { Provides specific guidance regarding } \\
\text { the adoption of Common Core State } \\
\text { Standards and the shift to personal } \\
\text { digital learning. }\end{array}$ & $\begin{array}{l}\text { Singel site, Reference } \\
\text { book, No experiment }\end{array}$ \\
\hline 3 & $\begin{array}{c}\text { Banyen, W.; } \\
\text { Viriyavejakul, C.; } \\
\text { Ratanaolarn, T. (2016) }\end{array}$ & universitas Thailand & Teascher ans student & Exsperimental & $\begin{array}{c}\text { International Journal of } \\
\text { Emerging Technologies in } \\
\text { Learning (iJET) }\end{array}$ & $\begin{array}{l}\text { educational technology; Edmodo; } \\
\text { information literacy; self-directed } \\
\text { learning; social learning network }\end{array}$ & $\begin{array}{l}\text { - Tool validity not } \\
\text { discussed }\end{array}$ \\
\hline 4 & Graham, C. R. (2013). & $\begin{array}{l}\text { The pennsylvania } \\
\text { state university }\end{array}$ & -Teacher and student & - & New York, NY: Routledge. & Distance education, Blended learning & $\begin{array}{l}\text { Singel site, Reference } \\
\text { book, No experiment }\end{array}$ \\
\hline 5 & $\begin{array}{l}\text { Hamilton, J., \& Tee, S. } \\
\text { (2013) }\end{array}$ & & Teacher and student & Quantitative & Taylor \& Francis, 2013 & $\begin{array}{l}\text { Biggs, blended, flexible, student } \\
\text { learning outcomes, tertiary teaching } \\
\text { modes, traditional }\end{array}$ & $\begin{array}{l}\text { Singel site, Learning } \\
\text { Method Comparison, } \\
\text { No experiment }\end{array}$ \\
\hline 6 & Hilliard, A. T. (2015). & - & Teacher and student & Qualitative & $\begin{array}{l}\text { Journal of International } \\
\text { Education Research }\end{array}$ & $\begin{array}{l}\text { Leadership, Blended Learning, } \\
\text { Expanding Globally, Faculty } \\
\text { Development, Views of Student } \\
\text { Learners, Policy and Evaluation }\end{array}$ & $\begin{array}{l}\text { - No power analysis, } \\
\text { Tool not validated } \\
\text { after, modification, } \\
\text { No experiment }\end{array}$ \\
\hline 7 & $\begin{array}{l}\text { Kintu, M. J., Zhu, C., \& } \\
\text { Kagambe, E. (2017). }\end{array}$ & - & 238 respondent student & Survey & $\begin{array}{c}\text { International Journal of } \\
\text { Educational Technology in } \\
\text { Higher Education }\end{array}$ & $\begin{array}{l}\text { Blended learning effectiveness, } \\
\text { Learner characteristics, Design } \\
\text { features, Learning outcomes, and } \\
\text { significant predictors }\end{array}$ & $\begin{array}{l}\text { All in the form of a } \\
\text { survey there were no } \\
\text { experiments, no } \\
\text { vocational data }\end{array}$ \\
\hline 8 & $\begin{array}{l}\text { Korr, Derwin, Greene, \& } \\
\text { Sokoloff,. (2012). }\end{array}$ & $\begin{array}{l}\text { Brandman } \\
\text { University }\end{array}$ & Student & $\begin{array}{l}\text { implementation of } \\
\text { blended learning }\end{array}$ & & $\begin{array}{l}\text { adult learning, andragogy, blended, } \\
\text { hybrid, instructional design }\end{array}$ & $\begin{array}{l}\text { no vocational data, } \\
\text { Singel site, No } \\
\text { experiment }\end{array}$ \\
\hline 9 & Poon \& Joanna. (2013). & $\begin{array}{l}\text { NTU undergraduate } \\
\text { SPUR }\end{array}$ & $\begin{array}{c}\text { interviews with } \\
\text { academics } \\
\text { conducted by an NTU } \\
\text { undergraduate SPUR } \\
\text { award recipient and a } \\
\text { questionnaire survey of } \\
\text { students. } \\
\end{array}$ & Survey & $\begin{array}{l}\text { MERLOT Journal of } \\
\text { Online Learning and } \\
\text { Teaching }\end{array}$ & $\begin{array}{l}\text { blended learning, hybrid learning, } \\
\text { institutional approach, student } \\
\text { learning experiences }\end{array}$ & $\begin{array}{l}\text { No educator data } \\
\text { collected, Tool } \\
\text { validity not discussed, } \\
\text { No power analysis. }\end{array}$ \\
\hline 10 & $\begin{array}{l}\text { Saliba, G., Lynnae, R., \& } \\
\text { Cortez, H. (2013) }\end{array}$ & Australia & -Teacher and student & - & $\begin{array}{l}\text { University of Western } \\
\text { Sydney }\end{array}$ & $\begin{array}{l}\text { Fundamental blended learning, } \\
\text { category blended learning }\end{array}$ & $\begin{array}{l}\text { Singel site, Reference } \\
\text { book, No power } \\
\text { analysis }\end{array}$ \\
\hline
\end{tabular}


Table 1 continuous

\begin{tabular}{|c|c|c|c|c|c|c|c|}
\hline 11 & Singh H 2003 & - & - & - & $\begin{array}{c}\text { Issue of Educational } \\
\text { Technology }\end{array}$ & $\begin{array}{l}\text { Blended learning, Effective Blended } \\
\text { Learning }\end{array}$ & $\begin{array}{c}\text { No educator data } \\
\text { collected, Tool } \\
\text { validity not discussed, } \\
\text { No power analysis, } \\
\text { No sample }\end{array}$ \\
\hline 12 & $\begin{array}{l}\text { Staker, H., \& Horn, M. } \\
\text { B. (2012) }\end{array}$ & Innosight institute & - & - & Innosight institute & $\begin{array}{l}\text { Blended-learning taxonomy. Model } \\
\text { Blended learning, category blended } \\
\text { learning, }\end{array}$ & $\begin{array}{c}\text { No educator data } \\
\text { collected, Tool } \\
\text { validity not discussed, } \\
\text { No power analysis, } \\
\text { No sample, Reference } \\
\text { book, Singel site }\end{array}$ \\
\hline 13 & $\begin{array}{c}\text { Graham, C. R., } \\
\text { Woodfield, W., \& } \\
\text { Harrison, J. B. (2013). }\end{array}$ & $\begin{array}{c}\text { Brigham Young } \\
\text { University, United } \\
\text { States }\end{array}$ & $\begin{array}{c}\text { Interviews with } \\
\text { administrators at } \\
\text { institutions of higher } \\
\text { learning }\end{array}$ & $\begin{array}{l}\text { Qualitative with } \\
\text { Interviews }\end{array}$ & $\begin{array}{l}\text { Internet and Higher } \\
\text { Education, } \\
\text { ELSEVIER }\end{array}$ & $\begin{array}{l}\text { Blended learning Hybrid courses } \\
\text { Institutional adoption Higher } \\
\text { education policy }\end{array}$ & $\begin{array}{l}\text { Tool validity not } \\
\text { discussed, no } \\
\text { statistical data, No } \\
\text { experiment } \\
\end{array}$ \\
\hline 14 & $\begin{array}{l}\text { McGee, P., \& Reis, A. } \\
\text { (2012). }\end{array}$ & Outside of class & $\begin{array}{c}\text { Literature } \\
\text { review/publication }\end{array}$ & $\begin{array}{l}\text { qualitative } \\
\text { meta-analysis design } \\
\text { to answer one } \\
\text { research question: }\end{array}$ & $\begin{array}{c}\text { Journal of Asynchronous } \\
\text { Learning Networks } \\
\text { ERIC }\end{array}$ & $\begin{array}{l}\text { blended, hybrid, course design, } \\
\text { pedagogy, best practices }\end{array}$ & $\begin{array}{c}\text { No educator data } \\
\text { collected, Tool } \\
\text { validity not discussed, } \\
\text { No power analysis, } \\
\text { No experiment }\end{array}$ \\
\hline 15 & $\begin{array}{c}\text { Verawadina, U., Jalinus, } \\
\text { N., Krismadinata, } \\
\text { Widya, R.N., \& Simeru, } \\
\text { A. (2020). }\end{array}$ & Indonesia & $\begin{array}{c}\text { a) Male and female } \\
\text { students b) Informatics } \\
\text { Engineering and } \\
\text { Automotive Education } \\
\text { Study Programs c) } \\
\text { Visual programming and } \\
\text { Autotronics Courses }\end{array}$ & $\begin{array}{c}\text { a quantitative } \\
\text { descriptive study. }\end{array}$ & $\begin{array}{c}\text { International Journal of } \\
\text { Innovation, Creativity, and } \\
\text { Change. } w\end{array}$ & $\begin{array}{l}\text { Needs assessment, e-learning, } \\
\text { blended learning. }\end{array}$ & Singel site \\
\hline 16 & $\begin{array}{c}\text { Verawardina, U., Asnur, } \\
\text { L., Lubis, A. L., \& } \\
\text { Hendriyani, Y. (2020). }\end{array}$ & Indonesia & $\begin{array}{c}\text { Literature } \\
\text { review/publication }\end{array}$ & $\begin{array}{l}\text { method of analysis } \\
\text { of literature ( }\end{array}$ & $\begin{array}{l}\text { Talent Development \& } \\
\text { Excellence }\end{array}$ & $\begin{array}{l}\text { Online Learning, Revolution } \\
\text { industry 4.0, COVID-19. }\end{array}$ & $\begin{array}{c}\text { Singel site, No } \\
\text { educator data } \\
\text { collected, Tool } \\
\text { validity not discussed, } \\
\text { No power analysis, } \\
\text { No experiment } \\
\end{array}$ \\
\hline 17 & $\begin{array}{l}\text { Verawardina, U., } \\
\text { Ramadhani, D., Susanti, } \\
\text { W., Lubis, A. L., \& } \\
\text { Simeru, A. (2020). }\end{array}$ & Indonesia & $\begin{array}{c}\text { Literature } \\
\text { review/publication }\end{array}$ & $\begin{array}{l}\text { method of analysis } \\
\text { of literature ( }\end{array}$ & $\begin{array}{l}\text { International Journal of } \\
\text { Psychosocial } \\
\text { Rehabilitation, }\end{array}$ & $\begin{array}{c}\text { XXI Century Learning, technology, } \\
\text { Mooc, online learning }\end{array}$ & $\begin{array}{c}\text { Singel site, No } \\
\text { educator data } \\
\text { collected, Tool } \\
\text { validity not discussed, } \\
\text { No power analysis, } \\
\text { No experiment } \\
\end{array}$ \\
\hline 18 & $\begin{array}{l}\text { Clark, R. C., \& Kwinn, } \\
\text { A. } 2007\end{array}$ & - & - & - & Publishser by Pfeiffer. & $\begin{array}{c}\text { The new virtual classroom, learning, } \\
\text { synchronous }\end{array}$ & $\begin{array}{l}\text { Singel site, Reference } \\
\text { book, No experiment }\end{array}$ \\
\hline 19 & $\begin{array}{l}\text { Allen, I. E., Seaman, J., } \\
\text { \& Garrett, R. (2007). }\end{array}$ & $\begin{array}{l}\text { the United States } \\
\text { America }\end{array}$ & - & - & $\begin{array}{l}\text { by Sloan-CTM } \\
\text { ERIC }\end{array}$ & $\begin{array}{l}\text { Blending, education, The Extent, and } \\
\text { Promise of Blended Education }\end{array}$ & Reference book \\
\hline
\end{tabular}


Table 1 continuous

\begin{tabular}{|c|c|c|c|c|c|c|c|}
\hline 20 & Caner, M. (2012). & - & $\begin{array}{l}\text { Literature } \\
\text { review/publication }\end{array}$ & $\begin{array}{l}\text { method of analysis } \\
\text { of literature ( }\end{array}$ & researchgate.net & $\begin{array}{c}\text { The Definition of Blended Learning, } \\
\text { Higher Education }\end{array}$ & $\begin{array}{c}\text { not on-site, unclear } \\
\text { data collection, } \\
\text { unclear validity, } \\
\text { unclear data analysis, } \\
\text { No experiment } \\
\end{array}$ \\
\hline 21 & $\begin{array}{l}\text { Dewi, Ciptayani, } \\
\text { Surjono, \& Priyanto. } \\
\text { (2018) }\end{array}$ & Indonesia & $\begin{array}{l}\text { Politeknik Negeri Bali } \\
\text { (PNB), Indonesia }\end{array}$ & $\begin{array}{l}\text { The study is done by } \\
\text { research and } \\
\text { development } \\
\text { approach to develop } \\
\text { a blended learning } \\
\text { model in Vocational }\end{array}$ & $\begin{array}{c}\text { TOJET: The Turkish } \\
\text { Online Journal of } \\
\text { Educational Technology } \\
\text { ERIC }\end{array}$ & $\begin{array}{l}\text { Modeling Vocational Blended } \\
\text { Learning, Digital Learning Now } \\
\text { Framework, Vocational }\end{array}$ & $\begin{array}{l}\text { No specific sample, } \\
\text { No experiment }\end{array}$ \\
\hline 22 & $\begin{array}{l}\text { Graham, C., Henrie, C., y } \\
\text { Gibbons, A. (2014). }\end{array}$ & - & - & - & NY. P.: Routledge. & $\begin{array}{c}\text { Model blended learning, theory } \\
\text { blended learning }\end{array}$ & Reference book \\
\hline 23 & $\begin{array}{l}\text { Allen, I. E., \& Seaman, J. } \\
(2003) .\end{array}$ & America & Public, student, teacher & $\begin{array}{c}\text { Survey } \\
\text { Study literature } \\
\end{array}$ & ERIC & $\begin{array}{c}\text { The Quality and Extent of Online } \\
\text { Education }\end{array}$ & $\begin{array}{r}\text { Singel Site, Reference } \\
\text { book, No experiment }\end{array}$ \\
\hline 24 & $\begin{array}{l}\text { Chew, Esyin \& Jones, } \\
\text { Norah \& Turner, David. } \\
\text { (2018) }\end{array}$ & $\begin{array}{l}\text { The United } \\
\text { Kingdom, }\end{array}$ & - & $\begin{array}{l}\text { It is an educational } \\
\text { and critical review }\end{array}$ & researchgate.net & $\begin{array}{c}\text { Hybrid Learning, Blended learning, } \\
\text { Educational Theory, Higher } \\
\text { Education }\end{array}$ & $\begin{array}{l}\text { not on site, unclear } \\
\text { data collection, } \\
\text { unclear validity, } \\
\text { unclear data analysis }\end{array}$ \\
\hline 25 & $\begin{array}{l}\text { Owston, R., \& York, D. } \\
\text { N. (2018). }\end{array}$ & university in Canada & $\begin{array}{c}\text { Students in } 20 \\
\text { undergraduate courses o }\end{array}$ & Survei & $\begin{array}{l}\text { http://www.yorku.ca/ } \\
\text { ELSEVIER } \\
\text { The Internet and Higher } \\
\text { Education } \\
\end{array}$ & $\begin{array}{l}\text { Blending learning, Learning } \\
\text { environment design Teaching }\end{array}$ & No experiment \\
\hline 26 & Wena, M., 2010. & Indonesia & - & - & Bumi Aksara & innovative learning, learning strategy & $\begin{array}{c}\text { References book, No } \\
\text { power analysis, No } \\
\text { experiment }\end{array}$ \\
\hline 27 & $\begin{array}{l}\text { Kamsin, Amirrudin. } \\
2005 .\end{array}$ & Malaysia & $\begin{array}{c}\text { Participants were } 78 \\
\text { students, and one female } \\
\text { lecturer }\end{array}$ & $\begin{array}{l}\text { A survey was } \\
\text { conducted for the } \\
\text { students. }\end{array}$ & $\begin{array}{c}\text { http://www.journal.au.edu/ } \\
\text { International Journal of The } \\
\text { Computer, the Internet and } \\
\text { Management }\end{array}$ & $\begin{array}{l}\text { E-learning, Conventional learning, } \\
\text { knowledge sharing, }\end{array}$ & No experiment \\
\hline 28 & $\begin{array}{l}\text { Seninil, Mochamad Bruri } \\
\text { Triyono, Asnul Dahar } \\
\text { Minghat, Herman Dwi } \\
\text { Surjono, Siti Salina } \\
\text { Mustakim . 2018. }\end{array}$ & Indonesia & $\begin{array}{l}\text { vocational high school } \\
\text { (SMK) }\end{array}$ & Study literature & $\begin{array}{l}\text { International Journal of } \\
\text { Engineering \& Technology }\end{array}$ & $\begin{array}{l}\text { E-learning; Technical and vocational } \\
\text { education; Vocational high school. }\end{array}$ & $\begin{array}{c}\text { Singel site, Tool } \\
\text { validity not discussed, } \\
\text { No power analysis, } \\
\text { No experimen }\end{array}$ \\
\hline 29 & $\begin{array}{c}\text { Syarifah, Mufarrahatus } \\
\text { dan Handayani, Hany. } \\
2019 .\end{array}$ & Indonesia & $\begin{array}{l}43 \text { elementary school } \\
\text { teachers }\end{array}$ & Descriptive & $\begin{array}{c}\text { unri.ac.id } \\
\text { Journal of Teaching and } \\
\text { Learning in Elementary } \\
\text { Education (JTLEE) } \\
\end{array}$ & $\begin{array}{l}\text { E-learning, blended learning, } \\
\text { teachers' conception }\end{array}$ & $\begin{array}{c}\text { Tool validity not } \\
\text { discussed, No } \\
\text { experiment, No power } \\
\text { analysis } \\
\end{array}$ \\
\hline
\end{tabular}


Table 1 continuous

\begin{tabular}{|c|c|c|c|c|c|c|c|}
\hline 30 & $\begin{array}{l}\text { Bjørg F. Oftedal, Kristin } \\
\text { H. Urstad, Venche } \\
\text { Hvidsten, and Brynjar } \\
\text { Foss. } 2015 .\end{array}$ & - & $\begin{array}{l}\text { all nursing students } \\
\text { enrolled in } 2009 \text { and } \\
2010 \text { attending the } \\
\text { three-year on-campus } \\
\text { program (OCP) or the } \\
\text { four-year blended } \\
\text { learning program (BLP) }\end{array}$ & $\begin{array}{l}\text { The study had a } \\
\text { descriptive } \\
\text { quantitative design. }\end{array}$ & $\begin{array}{l}\text { International Journal of } \\
\text { Learning, Teaching and } \\
\text { Educational Research }\end{array}$ & $\begin{array}{l}\text { Blended learning; Bachelor in } \\
\text { nursing; Learning outcome; } \\
\text { Quantitative method }\end{array}$ & $\begin{array}{l}\text { Singel site, Tool } \\
\text { validity not discussed, } \\
\text { No experiment. }\end{array}$ \\
\hline 31 & Mestan, Kamran, 2009. & La Trobe University & Student 5,477, & $\begin{array}{l}\text { The case study } \\
\text { applied mixed } \\
\text { methods }\end{array}$ & $\begin{array}{l}\text { Australasian Journal of } \\
\text { Educational Technology }\end{array}$ & $\begin{array}{l}\text { Create a fine blend, blended } \\
\text { learning, An examination of } \\
\text { institutional } \\
\end{array}$ & $\begin{array}{c}\text { Singel site, Tool } \\
\text { validity not discussed, } \\
\text { No experiment }\end{array}$ \\
\hline 32 & $\begin{array}{c}\text { Menon, Sujatha } \\
\text { Aravindakshan. } 2019 .\end{array}$ & $\begin{array}{l}\text { Jazan University, } \\
\text { Kingdom of Saudi } \\
\text { Arabia }\end{array}$ & Teacher and student & Study literature & $\begin{array}{c}\text { Researchgate, } \\
\text { Neliti } \\
\text { International Journal of } \\
\text { Linguistics, Literature, and } \\
\text { Translation (IJLLT) } \\
\end{array}$ & $\begin{array}{l}\text { Teaching/ learning process, } \\
\text { learners, BookWidgets, } \\
\text { technology, online materials, } \\
\text { blended learning }\end{array}$ & $\begin{array}{c}\text { Tool validity not } \\
\text { discussed, No } \\
\text { experiment, No power } \\
\text { analysis }\end{array}$ \\
\hline 33 & Chairman, U.2017 & Indonesia & - & $\begin{array}{l}\text { Study literature, } \\
\text { survey }\end{array}$ & Researchgate, & $\begin{array}{l}\text { Online learning, blended } \\
\text { learning. Hinger education }\end{array}$ & $\begin{array}{c}\text { Reference book, } \\
\text { Singel site, Tool } \\
\text { validity not discussed, } \\
\text { No experiment } \\
\end{array}$ \\
\hline 34 & $\begin{array}{l}\text { Coronel, C., \& Tan, D. } \\
\text { A. (2019) }\end{array}$ & $\begin{array}{l}\text { Valencia City, } \\
\text { Bukidnon. }\end{array}$ & $\begin{array}{l}\text { The experimental group } \\
\text { were twenty-five (25) } \\
\text { students, and the control } \\
\text { group was twenty-eight } \\
\text { (28) }\end{array}$ & Experimental & ERIC & $\begin{array}{l}\text { mathematics performance, } \\
\text { self-blend approach, } 21 \mathrm{st} \\
\text { century skills }\end{array}$ & $\begin{array}{l}\text { Tool validity not, } \\
\text { discussed }\end{array}$ \\
\hline 35 & $\begin{array}{l}\text { Alnoori, B. A., \& Obaid, } \\
\text { S. S. (2017). }\end{array}$ & - & $\begin{array}{l}\text { The sample consists of } \\
20 \text { students }\end{array}$ & $\begin{array}{l}\text { The current study } \\
\text { design consists of } \\
\text { two tests: pre-test } \\
\text { and post-test. }\end{array}$ & $\begin{array}{l}\text { semanticscholar.org } \\
\text { International Journal of } \\
\text { Language Academy }\end{array}$ & $\begin{array}{l}\text { blended Learning, reading } \\
\text { Skills, secondary Schools, } \\
\text { technology, method }\end{array}$ & $\begin{array}{l}\text { Littel example, Singel } \\
\text { site, Tool validity not } \\
\text { discussed. }\end{array}$ \\
\hline 36 & Kocour, N. (2019) & $\begin{array}{l}\text { Northwestern } \\
\text { College - Orange } \\
\text { City }\end{array}$ & $\begin{array}{l}\text { Thirty-five participants } \\
\text { in the study, ranging in } \\
\text { age from three to } \\
\text { five-year-olds. }\end{array}$ & $\begin{array}{l}\text { The purpose of this } \\
\text { study was to } \\
\text { determine the } \\
\text { relationship. }\end{array}$ & $\begin{array}{c}\text { [PDF] nwciowa.edu } \\
\text { https://nwcommons.nwciowa.e } \\
\mathrm{du} /\end{array}$ & $\begin{array}{l}\text { blended learning, student } \\
\text { engagement, early childhood }\end{array}$ & $\begin{array}{c}\text { Singel site, Tool } \\
\text { validity not discussed, } \\
\text { No experiment }\end{array}$ \\
\hline 37 & $\begin{array}{c}\text { Asia, A., \& Wekke, I. S. } \\
\text { (2019). }\end{array}$ & Indonesia & Lecture & $\begin{array}{l}\text { This study, using the } \\
\text { case study method, }\end{array}$ & $\begin{array}{c}\text { Researchgate. } \\
\text { Universal Journal of } \\
\text { Educational Research (7) }\end{array}$ & $\begin{array}{l}\text { Participation, Blended Learning, } \\
\text { Industrial Revolution } 4.0\end{array}$ & $\begin{array}{c}\text { Tool validity not } \\
\text { discussed, Tool } \\
\text { validity not discussed, } \\
\text { No experiment } \\
\end{array}$ \\
\hline 38 & $\begin{array}{l}\text { Bliuc, A. M., Casey, G., } \\
\text { Bachfischer, A., } \\
\text { Goodyear, P., \& Ellis, R. } \\
\text { A. (2012). }\end{array}$ & Australia & Teacher & $\begin{array}{c}\text { Teachers' } \\
\text { conceptions and } \\
\text { their approaches } \\
\text { were explored by } \\
\text { asking participants } \\
\text { to respond to the } \\
\text { questions. }\end{array}$ & $\begin{array}{c}\text { https://www.researchgate.net/ } \\
\text { Aust. Educ. Res. }\end{array}$ & $\begin{array}{l}\text { Blended learning, Blended } \\
\text { learning design, Conceptions, } \\
\text { Approaches, Vocational } \\
\text { education, Teacher experiences }\end{array}$ & $\begin{array}{l}\text { Tool validity not } \\
\text { discussed, No } \\
\text { experiment. }\end{array}$ \\
\hline
\end{tabular}


Table 1 continuous

\begin{tabular}{|c|c|c|c|c|c|c|c|}
\hline 39 & Şahin, M. (2010). & Turkey. & $\begin{array}{l}\text { The study group consists } \\
\text { of } 56 \text { students, } 28 \text { of } \\
\text { whom are of the control } \\
\text { group, and the other } 28 \\
\text { students are } \\
\text { experimental group. }\end{array}$ & $\begin{array}{l}\text { The research } \\
\text { involved an } \\
\text { experimental model. }\end{array}$ & $\begin{array}{l}\text { https://academicjournals.org/ } \\
\text { International Journal of } \\
\text { Vocational and Technical } \\
\text { Education }\end{array}$ & $\begin{array}{l}\text { Blended learning, vocational } \\
\text { education, experimental study. }\end{array}$ & $\begin{array}{l}\text { Tool validity not } \\
\text { discussed }\end{array}$ \\
\hline 40 & $\begin{array}{l}\text { Azhar, M., Mustapa, S., } \\
\text { Ibrahim, M., \& Yusoff, } \\
\text { A. (2015). }\end{array}$ & Malaysia & $\begin{array}{l}\text { vocational students and } \\
\text { teachers in VC under the } \\
\text { management of the } \\
\text { Ministry of Education } \\
\text { (MOE). }\end{array}$ & $\begin{array}{l}\text { This study aims to } \\
\text { investigate the use of } \\
\text { e-learning using } \\
\text { Web } 2.0 \\
\text { technology-based } \\
\text { applications. }\end{array}$ & $\begin{array}{c}\text { Ademia.edu } \\
\text { Conference proceedings of } \\
\text { the } 4 \text { th World Congress on } \\
\text { TVET } 2014\end{array}$ & $\begin{array}{c}\text { Blended learning; Web 2.0; } \\
\text { Vocational; }\end{array}$ & $\begin{array}{l}\text { Singel site, Tool } \\
\text { validity not discussed, } \\
\text { No power analysis, } \\
\text { No experiment }\end{array}$ \\
\hline 41 & $\begin{array}{c}\text { Patmanthara, S., \& } \\
\text { Hidayat, W. N. (2018, } \\
\text { June). }\end{array}$ & Indonesia & $\begin{array}{l}\text { with } 3 \text { practical classes } \\
\text { and three control classes. }\end{array}$ & $\begin{array}{c}\text { The research method } \\
\text { used was } \\
\text { Pretest-Posttest } \\
\text { Control Group } \\
\text { Design. } \\
\end{array}$ & $\begin{array}{c}\text { IOP } \\
\text { 2nd International Conference } \\
\text { on Statistics, Mathematics, } \\
\text { Teaching, and Research }\end{array}$ & $\begin{array}{c}\text { Vocational High School Students, } \\
\text { Digital Literacy Blended Learning } \\
\text { Model, }\end{array}$ & Singel site \\
\hline 42 & $\begin{array}{l}\text { Machumu, H. J., Zhu, C., } \\
\text { \& Sesabo, J. K. (2016). }\end{array}$ & Tanzania & $\begin{array}{l}\text { interview and focus } \\
\text { group discussion was } \\
\text { conducted with } 15 \text { VET } \\
\text { educators in three VET } \\
\text { colleges in both } \\
\text { Morogoro } \\
\end{array}$ & $\begin{array}{l}\text { This is a study } \\
\text { employed a } \\
\text { qualitative single } \\
\text { case study design. }\end{array}$ & $\begin{array}{c}\text { International Journal of } \\
\text { Multicultural and } \\
\text { Multireligious Understanding } \\
\text { (IJMMU) }\end{array}$ & $\begin{array}{c}\text { Blended Learning; Vocational } \\
\text { Education and Training; Learning } \\
\text { Technologies and } \\
\text { Professional Development; } \\
\text { Tanzania }\end{array}$ & $\begin{array}{l}\text { Singel site, Tool } \\
\text { validity not discussed, } \\
\text { No experiment }\end{array}$ \\
\hline 43 & $\begin{array}{l}\text { El Hajji, M., Bouzaidi, } \\
\text { E., Drissi, R., Douzi, H., } \\
\text { \& Khouya, E. H. (2016). }\end{array}$ & Maroko & Teacher and student & Experiment & $\begin{array}{c}\text { ERIC } \\
\text { Journal of Education and } \\
\text { Practice } \\
\end{array}$ & $\begin{array}{l}\text { Blended learning, flipped learning, } \\
\text { vocational work-linked Training. }\end{array}$ & $\begin{array}{l}\text { Tool validity not } \\
\text { discussed }\end{array}$ \\
\hline 44 & $\begin{array}{l}\text { Chang, C. C., Shu, K. M., } \\
\text { Liang, C., Tseng, J. S., \& } \\
\text { Hsu, Y. S. (2014 }\end{array}$ & Taiwan & $\begin{array}{l}\text { the experimental group } \\
33 \text {, and control group } 32 \text {, }\end{array}$ & $\begin{array}{l}\text { The pretest-posttest } \\
\text { nonequivalent-group } \\
\text { quasi-experimental }\end{array}$ & $\begin{array}{l}\text { International Review of } \\
\text { Research in Open and } \\
\text { Distributed Learning }\end{array}$ & $\begin{array}{c}\text { Blended e-learning; } \\
\text { self-assessment; electrical } \\
\text { machinery; learning performance. }\end{array}$ & - \\
\hline 45 & $\begin{array}{c}\text { Deechai, W., } \\
\text { Sovajassatakul, T., \& } \\
\text { Petsangsri, S. (2019). }\end{array}$ & Thailand & $\begin{array}{l}\text { random sampling } 450 \\
\text { (student dan teacher) }\end{array}$ & evaluation & $\begin{array}{l}\text { Mediterranean Journal of } \\
\text { Social Sciences }\end{array}$ & $\begin{array}{c}\text { Needs evaluation, Blended } \\
\text { learning development, Critical } \\
\text { thinking skills, Vocational } \\
\text { students, Teachers at vocational } \\
\text { level }\end{array}$ & $\begin{array}{l}\text { Singel site, No } \\
\text { experiment }\end{array}$ \\
\hline
\end{tabular}


Based on table 1 regarding summary of qualitative studies, a meta-analysis has been carried out on the publication, then the Limitations have been seen

The results obtained are considered that Blended learning is an activity that takes place in two different areas, namely in person and online. Locally designed mixed learning should fit the environment for both teachers and students [30].

Thus, combining online and offline learning requires a precise proportion of application. The proportion of learning using information technology can be seen in the classifications in integrated face-to-face learning \& online can be see table 2 .

Table 2. Porpation Blended learning

\begin{tabular}{|c|c|l|}
\hline $\begin{array}{c}\text { Online } \\
\text { proportion }\end{array}$ & $\begin{array}{c}\text { Learning } \\
\text { Type }\end{array}$ & \multicolumn{1}{|c|}{ Details } \\
\hline $0 \%$ & Traditional & $\begin{array}{l}\text { Learning without using online } \\
\text { technology, teaching material is } \\
\text { delivered in writing or orally. }\end{array}$ \\
\hline $1-29 \%$ & $\begin{array}{l}\text { Web } \\
\text { Facilitated }\end{array}$ & $\begin{array}{l}\text { Learning that uses web-based } \\
\text { technology to facilitate face-to-face } \\
\text { learning (learning is enriched with } \\
\text { internet access). Usually, use a } \\
\text { learning management system and a } \\
\text { website to post syllabus and } \\
\text { assignments. }\end{array}$ \\
\hline $30-79 \%$ & Blended & $\begin{array}{l}\text { Learning that combines online and } \\
\text { face to face. Content is sent online, } \\
\text { usually discussion online, several } \\
\text { face-to-face. }\end{array}$ \\
\hline$>80 \%$ & Online & $\begin{array}{l}\text { I am learning where most or all } \\
\text { material is sent online. Do not have } \\
\text { a face to face meeting. }\end{array}$ \\
\hline
\end{tabular}

Source: [21][22][31]

Based on table 2, it can be seen that the proportion of blended learning is in the range of $30-79 \%$. Regarding the proportion of online activity, it appears that there is a relationship between the proportion of mixed and perceptions that result in Mixed Moderate (36\% - 40\% online) and High ( $50 \%$ online) and High (50\% online) tend to have the most positive perceptions of blended learning, compared to Low proportion ( $27 \%$ - $30 \%$ online) [32]. The result is in line with government policies that allow universities to implement blended learning.

Several universities in Indonesia have implemented blended learning with a range of $50 \%$ online and $50 \%$ offline. And also $60 \%$ enrolled in college offline and the others studied online. These proxies can adapt to individual courses and other considerations. Meanwhile, according to Saeman, theoretical subjects for online proportions are certainly more dominant or whereas practical subjects use offline learning more. The combination of face-to-face and online was done in a complementary manner, while activities and online communication and face-to-face adjustments are needed. In results, teachers must be able to identify the characteristics of the courses applied.

In general, e-learning can be used as a supplement, complement, and substitute.

The functions of e-learning are 1). E-learning can be used as an addition so that students get additional knowledge, 2). Complementary materials, 3). can be a substitute for learning in the classroom [33].

Online learning is better as a supplement for classroom teaching, and the level of e-learning as a classroom teaching supplement can be determined according to the ability of institutions and developers and the ability of the users to adapt [34]. The use of e-learning serves as a complement in the effectiveness of learning in the classroom, which is able to increase knowledge about the material as well as students' learning motivation in vocational schools [35]. Blended learning is developed to replace lectures and provide support for and complete courses [36].

Blended Learning can complement direct learning [37]. The function of e-learning itself in learning is only as a complement. Blended learning plays more of a supplementary or a complementary role. Moreover, there is the existence of synchronous and asynchronous learning settings that contain face-to-face learning in class and online makes learning can happen flexibly anywhere and anytime with being connected to each other in digital form, and in which there are also collaborative discussions, projects, interactions, evaluations and various learning resources that can stimulate students [23].

The results of the study of [38] suggest that ongoing professional developments, institutions must support online design facilities, blended learning in vocational. According to [39], many programs at tertiary institutions use blended learning covering.

To be able to carry out blended learning well, it is necessary to make policies, plans, resources (such as human resources, equipment, technology), set of schedules, and support system initiatives to be successful. In order to carry out development programs, universities must also educate academics, for example instructors in using blended learning, redesigning online courses to be effective, and effective use of technology [40].

Factors considered in implementing blended learning are the ability to combine instructional modalities or media, the combination of online and face-to-face learning [25]. There are factors for creating a blended learning environment, including institutional, pedagogical, technology, interface design, evaluation, management, resource support, and ethics [41]. One problem that must be taken into account is the cost for each technology needed in integrating blended learning in vocational education. Other findings showed that the most crucial determinant of blended learning would succeed in vocational education as well as the ability of teachers to design learning models. Teachers must increase capacity by developing the ability to take advantage of technology and accept technology learning culture so that they can be 
easily understood by students.

According to [42]; [6], there are four typologies of the level of implementation of blended learning:

1. Activity level. Blended learning activities combine elements of face-to-face with elements of communication that are mediated through computer devices.

2. Course level. Blended learning courses combine face-to-face activities with activities in virtual classes online and can be done from a specific time.

3. Training program level. Blended learning can be programmed to be done online both at the beginning and end of the program or combining online activities with internships or offering online learning plans for students with limited attendance.

4. Institutional level. In this case, the tertiary institution applies for a reduction of face-to-face time in class, arranges curriculum periods for one semester, and offers online learning plans.

The blended learning standards are focused on four standards: a). Mixed learning is designed with a learner-centered pedagogy principle. b). Assessment and mixed feedback, c). Student interaction and engagement is facilitated by online communication and network channels via the internet. d). Students have access to a variety of quality resources and support (Saliba., Lynnae, \& Cortez, 2013).

Therefore, the typology of standard application of blended learning and blended learning which contains several principles, can be used as a reference for vocational education in developing a blended learning model with existing standards. Blended learning frameworks and models in producing novelty blended learning models in visual programming subjects.

There are a framework and model of blended learning to carry out the learning process. Here are some frameworks sourced from relevant books and research:

\section{a. Khan's Octagonal Framework}

The Khan's Octagonal Framework is used for blended learning programs that can be used in educational institutions in general, both in vocational education and in other tertiary institutions, even in schools. Khan's Octagonal Framework can be seen in Figure 2.

Based on figure 2, the framework has eight dimensions, namely: institutional, in this case, institutions and tertiary institutions; pedagogical that includes elements of learning; technological regarding the use of technology such as computer devices, LMS, computer networks, and others; interface design regarding the appearance and interface design of blended learning that adjusts the needs of each institution; evaluation; management to manage the running of the blended learning program must be appropriately managed which involves various elements in the education institution; resource support related to resources such as human resources and others; and requires ethical such as learning culture and attitude.

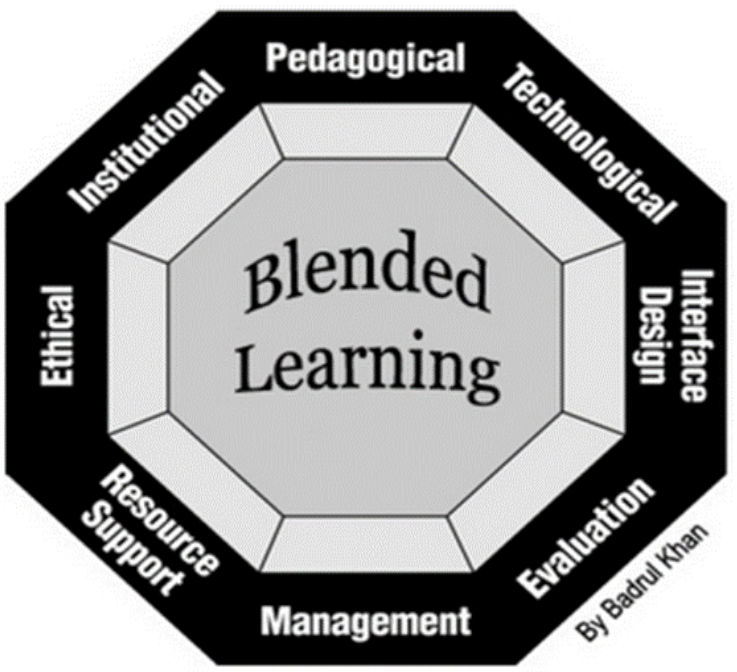

Figure 2. Khan's Octagonal Framework [44]

\section{b. Blended Learning Guide 3}

This blended learning model is in accordance with the digital learning framework used in education. The stages of creating conditions for success, planning, implementing, and improving can be seen in Figure 3.

\begin{tabular}{|l|l|l|l|}
\hline \multicolumn{1}{|c|}{ CREATE } \\
CONDITIONS \\
FOR SUCCESS
\end{tabular}

Figure 3. Framework of Blended Learning Guide 3 [45]

Digital learning framework guide 3 describes four phases starting from creating the conditions for success, planning, implementing, and improving.

In the first phase, the conditions for success are created, in which the shift in blended learning varies; need to build support, set academic goals, and need funding to make these learning changes. In the second phase, the application of the blended learning model needs to be planned which requires good planning. Preparing a good plan can be done by answering questions in six primary areas, such as a). strategies and timelines, b) school models, c) platforms and content, d) devices, e) staffing and development plans, g) improvement, and impact measurement.

The third stage is implementation which includes 
infrastructure, integration, professional development, support by paying attention to institutional culture, communication needs, and implementation success factors as well as considering how blended learning is implemented. It is important to remember that the overall goal of this shift to blended learning concerns teaching, learning, and design. The last phase is continuous improvement. Teachers and administrators in implementing blended learning must be able to make continuous improvements. This phase includes learning lessons, changes in size to measure changes in teaching and learning, future cultural innovations offering improvements in learning, developing multi-budget, and monitoring multi-year budgets [45].

\section{c. Vocational Blended Learning Modeling}

One of the developments of blended learning in vocational is Vocational Blended Learning (VBL) modeling. The results showed that in order to determine the learning model, it needs to consider the suitability of the educational model, techniques, teaching methods, facilities, and infrastructure. The framework of Vocational can be seen in Figure 4.
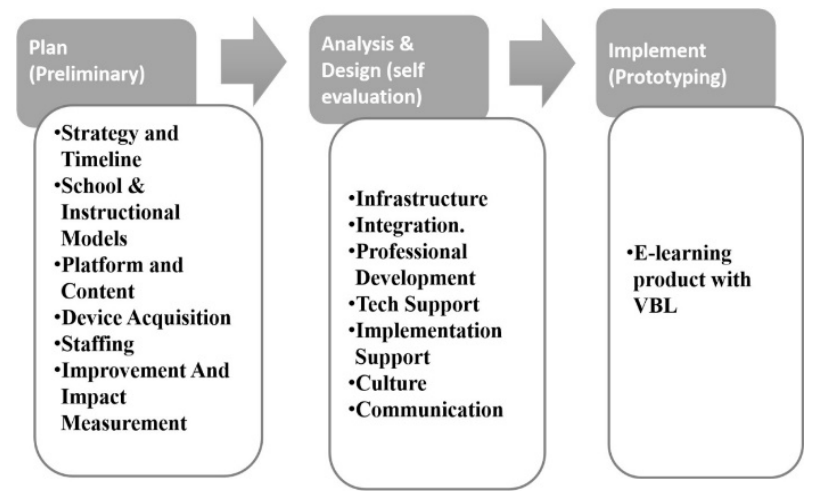

Figure 4. A framework of Vocational Blended Learning Modeling [46]

A framework of Vocational Blended Learning Modelin, describes three phases starting from plan, analysis, and design or self-evaluation and prototyping as the implementation phase as shown in figure 4. This framework requires 1) Infrastructure (technical) to measure the availability of information system hardware such as computer and server bandwidth. 2) Integration between content and the ICT learning process.

The development of professionalism and the successful application of blended learning depends on the abilities of human resources, lecturers, and students. It is also necessary to design lesson plans according to the needs of the model and select materials that are in accordance with the characteristics of vocational education. In the case of learning media, props are needed that can simulate actual working conditions.

Developing pedagogy in the use of information technology is instrumental in increasing teacher capacity. 4) Support (policy and financial), other factors that can support the successful use of blended learning are in the form of institutional policies and financial support.

The support provided can be in the form of strengthening infrastructure and also offering a budget for the development of teaching materials for lecturers, as well as related to policies in the use of blended learning such as guidelines that regulate the learning system between online and offline learning. 5). Culture, namely attitudes, readiness, and technological skills as a facilitator. Therefore, all factors significantly influence the successful use and development of information technology-based learning. The attitude towards acceptance of change is a factor that can encourage the successful implementation of blended learning. The blended learning model in education can be seen in Figure 5.

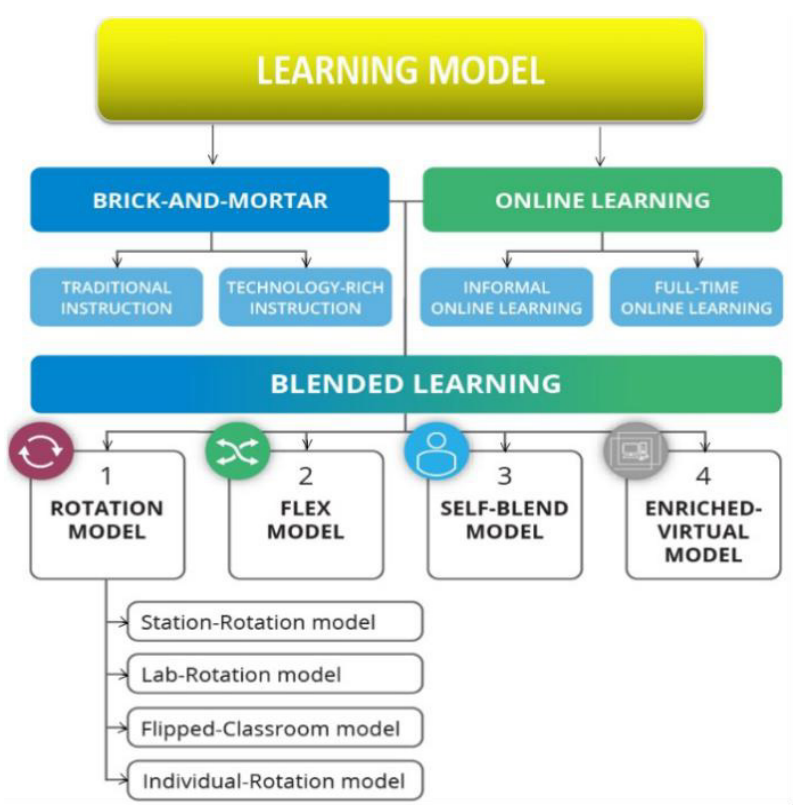

Figure 5. Blended learning in relation to other education practices [24]; [26]; [27], [28].

Based on Figure 5, blended learning in relation to other education practices. The Brick-and-mortar section is a traditional instruction and instruction using technology, both of which are combined learning to create a blended student learning experience. In traditional instruction, learning activities are centered on the teacher. Learning takes place using textbooks, lectures, and individual written assignments. Students are adjusted to their age and abilities. Technology-rich teaching, however, is learning that occurs with traditional teaching but uses digital enhancements such as electronic whiteboards, extensive access to internet devices, cameras, digital textbooks, the internet, and online lesson plans.

The online learning section consists of informal online learning, which is an educational program where students can use technology to study outside of the classroom at any time. For example, students use online videos to view course material. Full-time online learning is a structured education program where content and instructions are 
delivered via the internet. The students do not need to come to school. They can be remotely monitored online; however, at the time of the exam, students should come to school.

Based on the blended learning model categories that are composed of rotation models, flex models, self-blend models, enriched virtual models, the following are the explanation:

a. The rotation model is a program in a subject whose learning process can adjust to the schedule set by the lecturer. The learning process takes place through extensive group teaching, small group teaching, peer activities, group projects, individual tasks which can be done via computer. In this rotation model, there are several implementation settings as follows:

1) Rotation model: Students learn through a series of learning activities in class during specific scheduled periods. There is a rotation for online instructional activities. There are activities such as small group instruction as a whole, group projects, individual guidance, and assignments. Collaborative learning activities are carried out through small groups at each station.

2) Lab rotation: Students learn according to a predetermined schedule by working on individual instructions and dominant online learning done in the laboratory both throughout the class or in small groups.

3) Flipped Classroom: Students learn inside and outside the classroom with instructions and projects. Students individually access the content of teaching materials sent online with asynchronous strategies outside the classroom. The content of teaching material is accessed by students before face-to-face class meetings so that learning in the class evaluates individual understanding after learning online. The role of the lecturer is to control the student at any time when studying online in various places outside the classroom. This model contains the modalities of collaborative and project-based learning.

4) Individual Rotation: Students learn based on individual perceptions determined by the lecturer with the help of technology-based assessment equipment.

b. The flexible model is a learning program where content and instructions are delivered online, the teacher remains in place, while students study independently on a predetermined schedule. This model provides flexibility in learning, where the teacher can act as a guide. Other teacher-of-records offer flexible and adaptive offline support as needed through small group instruction activities, group projects, and individual mentoring. c. Self-Blend Model: Describes a scenario where students choose to take one or more lessons that all instructions are carried out online to complement their traditional courses. Teachers act as online instructors who are not on campus [24]. The Self-Blend Model is a fully individualized approach that allows students to choose to take one or more online courses to complete their traditional school catalog. Most part of the learning is done online, but students will still attend face-to-face classes. Self-blend is popular learning among students and teachers because it gives students the freedom to complete instructions that occur in class. Classes are combined with instructions and take part in online classes that help students hone their digital literacy skills, and this model is in line with entering the XXI century. The implementation of the self-blend discussion takes place to open up previous knowledge and concepts to students. Then the additional online activities provided, such as videos, worksheets, additional information about the topic, are given. This gives students the opportunity to take classes outside of what is already offered at their school. Individuals who attend classes in a traditional school environment choose to supplement their learning through online activities and videos.

d. The Enriched-Virtual Model is a learning implementation, where students are asked to be able to conduct face-to-face sessions with instructors, and then students can complete their assignments online. Students are not required to attend face-to-face classes. There are also face-to-face but optional classes [24].

The Digital Learning Now Framework can assist in planning and combining learning development in accordance with institutional conditions [46]. The blended learning model also applies to vocational education that regulates Vocational Blended Learning (VBL) by implementing a rotational learning model and supported by content developed by teachers [46].

Blended learning that is relevant to vocational, for example, is a polytechnic called a rotation model. Therefore, to implement this rotation model, the following aspects need to be considered:

a. Course content and analysis of learning units,

b. including content, coverage, topics, and semester credit;

c. Student analysis, such as students' educational background, age, gender, employment status;

d. Learning context analysis, on what kind of desired learning competition, must be discussed in depth.

e. Instructional analysis, regarding instructional materials grouped by interests, arranging tasks from easy to difficult; 
f. State the teaching objectives that can be arranged based on the results of the analysis of learning.

g. Create criteria test items. Test preparation should be based on the teaching objectives and test criteria should be established.

h. Create criteria test items. Test preparation is based on the teaching objectives and test criteria that must be made.

i. Choose a learning strategy. Teaching strategies can be established based on existing facilities [46].

It was concluded that the Digital Learning Now Framework could be used as a necessary foundation for developing blended learning in vocational education.

\section{Conclusions}

The original finding after conducting a meta-analysis of various published results was that blended learning is learning that combines offline learning as well as online learning.

This can create flexible learning, and can be done at any time in various places, also where there are interaction facilities and activities in it. The application of blended learning has its own proportion between offline and online. Before implementing blended learning in vocational education, it is necessary to pay attention to the things that are needed in the implementation of blended learning, such as the normal development of the blended learning model, application of topology, and also knowledge of the characteristics of the institution.

In particular, vocational education must adapt to vocational education, infrastructure, technology, learning, pedagogical principles, assessment of activities and baiting processes, interactions, resources, activities, infrastructure, culture, management and organization, ethics, and others. It is also necessary to develop blended learning stages so that they can be more systematic, starting with the stages of creating conditions for success, planning, implementing, and improving with various sub-stages. Blended learning can also increase the effectiveness of learning in vocational education as well as improve HOTS and skills.

Next, develop a blended learning model from Classifying K-12 Blended Learning that has four blended learning models to choose from, namely the rotation model (consisting of rotation model, lab rotation, and flipped individual classroom rotation), flex model, self-blend model, and enriched-virtual model. However, the rotation model is recommended for Vocational Blended Learning.

From the research results of this blended learning model, where vocational education applies blended learning, it can be suggested that it is not only limited to face-to-face and also online. There are several factors that must be considered in the development of blended learning, such as the proportion that must be correct. It is also hoped that the government, educational institutions, universities, and teachers to be able to develop a blended learning model using existing development stages must also go through the selection of a blended learning model from K-12 Blended Learning, so that blended learning can be found as a relevant vocational education model, practical, and effective to apply.

\section{REFERENCES}

[1] W. Banyen, C. Viriyavejakul, and T. Ratanaolarn, "A blended learning model for learning achievement enhancement of thai undergraduate students," Int. J. Emerg. Technol. Learn., vol. 11, no. 4, pp. 48-55, 2016, doi: 10.3991/ijet.v11i04.5325.

[2] W. Korr, J., Derwin, E. B., Greene, K., \& Sokoloff, "Transitioning an adult-serving university to a blended learning model," J. Contin. High. Educ., vol. 60, no. 1, pp. 2-11, 2012.

[3] A. M. Bliuc, G. Casey, A. Bachfischer, P. Goodyear, and R. A. Ellis, "Blended learning in vocational education: Teachers' conceptions of blended learning and their approaches to teaching and design," Aust. Educ. Res., vol. 39, no. 2, pp. 237-257, 2012, doi: 10.1007/s13384-012-0053-0.

[4] A. Verawadina, U., Jalinus, N., Krismadinata, Widya, R.N., \& Simeru, "Needs Assessment of E-Learning Vocational Education," Int. J. Innov. Creat. Chang., vol. 11, no. 4, pp. 262-274, 2020.

[5] M. Sahin, "Blended learning in vocational education: An experimental study," Int. J. Vocat. Tech. Educ., vol. 2, no. 6, pp. 95-101, 2010.

[6] C. R. Graham, W. Woodfield, and J. B. Harrison, "A framework for institutional adoption and implementation of blended learning in higher education," Internet High. Educ., vol. 18, pp. 4-14, 2013, doi: 10.1016/j.iheduc.2012.09.003.

[7] S. Hamilton, J., \& Tee, "Blended teaching and learning: a two-way systems approach.," High. Educ. Res. Dev., vol. 32, no. 5, pp. 748-764., 2013.

[8] A. L. Lubis, N. Jalinus, and R. Abdullah, "The Importance of Need Analysis for the Development of Co-PjBL Models on the Creative Products and Entrepreneurship Subject at the Vocational Level," Int. J. Adv. Sci. Technol., vol. 29, no. 7, pp. 985-993, 2020.

[9] A. Azhar, M., Mustapa, S., Ibrahim, M., \& Yusoff, "Engaging vocational college students through blended learning: Improving class attendance and participation," Procedia-Social Behav. Sci., vol. 204, pp. 127-135, 2015.

[10] F. Suryana, N. Jalinus, R. Rahmad, and R. Efendi, "Cooperative Project Based Learning Models in Programming Languages: A Proposed," Int. J. Adv. Sci. Technol., vol. 29, no. 06, pp. 1876-1886, 2020.

[11] J. Friadi, Ganefri, Ridwan, and R. Efendi, "Development of product based learning-teaching factory in the disruption era," Int. J. Adv. Sci. Technol., vol. 29, no. 6, pp. 1887-1898, 2020. 
[12] Ambiyar, Ganefri, Suryadimal, N. Jalinus, R. Efendi, and Jeprimansyah, "Development of work based learning (WBL) learning model in heat transfer courses," J. Phys. Conf. Ser. Ser., vol. 1481, 2020, doi: 10.1088/1742-6596/1481/1/0121 13.

[13] Ambiyar, R. Efendi, Y. Irawati, and Suryadimal, "Effectiveness e-authentic assessment in computer network course," J. Phys. Conf. Ser., vol. 1481, pp. 1-9, 2020, doi: 10.1088/1742-6596/1481/1/012131.

[14] R. Efendi and A. Yulastri, "Effectiveness of Collaborative Problem Based Learning Model of Learning Computer Network Courses," Proc. 5th UPI Int. Conf. Tech. Vocat. Educ. Train. (ICTVET 2018), vol. 299, no. Ictvet 2018, pp. 309-312, 2019, doi: 10.2991/ictvet-18.2019.70.

[15] R. Efendi, J. Jama, and A. Yulastri, "Development of Competency Based Learning Model in Learning Computer Networks," J. Phys. Conf. Ser., vol. 1387, no. 1, pp. 0-6, 2019, doi: 10.1088/1742-6596/1387/1/012109.

[16] R. Efendi, A. Yulastri, and Yusran, "Implementation Competency Based Learning Model of Learning Computer Network Courses at Vocational Education," J. Adv. Res. Dyn. Control Syst., vol. 11, no. 5, pp. 501-505, 2019.

[17] S. Patmanthara and W. N. Hidayat, "Improving Vocational High School Students Digital Literacy Skill through Blended Learning Model," J. Phys. Conf. Ser., vol. 1028, no. 1, 2018, doi: 10.1088/1742-6596/1028/1/012076.

[18] C. C. Chang, K. M. Shu, C. Liang, J. S. Tseng, and Y. S. Hsu, "Is blended e-learning as measured by an achievement test and self-assessment better than traditional classroom learning for vocational high school students?," Int. Rev. Res. Open Distance Learn., vol. 15, no. 2, pp. 213-231, 2014, doi: 10.19173/irrodl.v15i2.1708.

[19] W. Deechai, T. Sovajassatakul, and S. Petsangsri, "The Need for Blended Learning Development to Enhance the Critical Thinking of Thai Vocational Students," Mediterr. J. Soc. Sci., vol. 10, no. 1, pp. 131-140, 2019, doi: 10.2478/mjss-2019-0013.

[20] A. Clark, R. C., \& Kwinn, The new virtual classroom evidence-based guidelines for synchronous e-learning. San Francisco: Pfeiffer., 2007.

[21] I. E. Allen, J. Seaman, and R. Garrett, "Blending in: The extent and promise of blended education in the United States," Sloan Consort., pp. 1-29, 2007, doi: 10.1007/s00170-005-0274-8.

[22] M. Caner, "The definition of blended learning in higher education," Blended Learn. Environ. Adults Eval. Fram., no. April 2012, pp. 19-34, 2012, doi: 10.4018/978-1-4666-093 9-6.ch002.

[23] U. A. Chaeruman, Pedati Model Desain Sistem Pembelajaran Blended, no. January 2018. 2017.

[24] M. B. Staker, H., \& Horn, "Classifying K-12 Blended Learning," Innosight Institute., 2012. https://files.eric.ed.go v/fulltext/ED535180.pdf.

[25] A. Graham, C., Henrie, C., y Gibbons, "Developing models and Theory for Blended Learning Research. En A. Picciano, C. Dziuban, y C. Graham (Eds.), Research Perspectives in Blended Learning," Res. Perspect., vol. 2, pp. 13-33, 2014.
[26] B. Saadoon, M. Alnoori, and S. A. Obaid, "International Journal of Language Academy THE EFFECTIVENESS OF 50-50 BLENDED SKILLS IN ESL CLASSROOM," International J. Lang. Acad., vol. 5, no. December, pp. 288303, 2017.

[27] N. Kocour, How Blended Learning Impacts Student Engagement in an Early Childhood Classroom. Orange City: Northwestern College, 2019.

[28] A. Shamad and I. S. Wekke, "Lecturers' participation in applying blended learning in islamic higher education in Indonesia," Univers. J. Educ. Res., vol. 7, no. 12, pp. 2604 2608, 2019, doi: 10.13189/ujer.2019.071207.

[29] E. Kintu, M. J., Zhu, C., \& Kagambe, "Blended learning effectiveness: The relationship between student characteristics, design features and outcomes: Revista de universidad y sociedad del conocimiento," Int. J. Educ. Technol. High. Educ., vol. 14, pp. 1-20, 2017.

[30] H. J. Machumu, C. Zhu, and J. K. Sesabo, "Blended Learning in the Vocational Education and Training System in Tanzania: Understanding Vocational Educators' Perceptions," Int. J. Multicult. Multireligious Underst., vol. 3, no. 2, p. 30, 2016, doi: 10.18415/ijmmu.v3i2.46.

[31] D. Chew, Esyin \& Jones, Norah \& Turner, "Critical Review of the Blended Learning Models Based on Maslow's and Vygotsky's Educational Theory.," 2018.

[32] R. Owston and D. N. York, "The nagging question when designing blended courses: Does the proportion of time devoted to online activities matter?," Internet High. Educ., vol. 36, no. September 2017, pp. 22-32, 2018, doi: 10.1016/j.iheduc.2017.09.001.

[33] M. Wena, Strategi pembelajaran inovatif kontemporer. Jakarta: Bumi Aksara, 2010.

[34] K. Amirrudin, "Is E-Learning the solution and substitute for conventional learning?," Int. J. Comput. Internet Manag., vol. 13 , no. 3,2005 , pp. 79-89, 2005.

[35] S. S. M. Seninil, Mochamad Bruri Triyono, Asnul Dahar Minghat, Herman Dwi Surjono, "E-Learning Model for Technical and Vocational Education: In Vocational High School,” Int. J. Eng. Technol., vol. 7, pp. 526-529, 2018.

[36] B. F. Oftedal, K. H. Urstad, V. Hvidsten, and B. Foss, "Blended VS On-Campus Learning: A Study of Exam Results in the Bachelor Degree in Nursing," IJLTER, Int. J. Learn. Teach. Educ. Res., vol. 11, no. 3, pp. 59-68, 2015, [Online]. Available: http://ijlter.org/index.php/ijlter/article/ view/319.

[37] K. Mestan, "Create a fine blend: An examination of institutional transition to blended learning," Australas. J. Educ. Technol., vol. 35, no. 1, pp. 70-84, 2019, doi: 10.14742/ajet.3216

[38] M. Hajji, R. D. Bouzaidi, H. Douzi, and E. H. Khouya, "New blended learning strategy based on flipped-learning for vocational work-linked training," J. Educ. Pract., vol. 7, no. 36, pp. 126-130, 2016, [Online]. Available: http://files.eric.ed.gov/fulltext/EJ1126478.pdf.

[39] A. T. Hilliard, "Global blended learning practices for teaching and learning, leadership, and professional development," J. Int. Educ. Res., vol. 11, no. 3, pp. 179-n/a, 
2015.

[40] \& J. Poon, "Blended learning: an institutional approach for enh y.ancing students' Learning Experiences," MERLOT J. Online Learn. Teaching.

[41] Khan. Singh, "Building Effective Blended Learning Programs," Educ. Technol. Mag. Manag. Chang. Educ., vol. 43, no. 6, pp. 51-54, 2003.

[42] Graham CR, "Blended learning systems: definition, current trends, and future directions In C. Bonk and C. Graham (Eds.) the handbook of blended learning," Glob. Perspect. local Des. San Fr. Pfeiffer, 2006.

[43] H. Saliba, G., Lynnae, R., \& Cortez, "The Fundamentals of Blended Learning. Learning and Teaching Unit University of Western Sidney.," 2013.

[44] B. Khan, Managing e-learning: design delivery, implementation and evaluation. Hershey PA: Information Science Publishing, 2005.

[45] J. Bailey, C. Schneider, and T. Vander Ark, "Navigating the digital shift: Implementation strategies for blended and online learning," Digit. Learn. Now!, a Natl. Initiat. under Found. Excell. Educ., p. 253, 2013.

[46] K. C. Dewi, P. I. Ciptayani, and H. D. Surjono, "Modeling Vocational Blended Learning Based on Digital Learning Now Framework.," Turkish Online J. Educ. Technol. TOJET, vol. 17, no. 2, pp. 89-96, 2018.

[47] Novaliendry, D., Darmi, R., Hendriyani, Y., Nor, M., \& Azman, A. (2020). Smart Learning Media Based on Android Technology. International Journal of Innovation, Creativity and Change., 12(11), 715-735.

[48] D. Novaliendry, Y. Hendriyani, C-H. Yang, \& H. Hamimi, 2015, The Optimized K-Means Clustering Algorithms to Analyezed the Budget Revenue Expenditure in Padang. Proceeding of International Conference on Electrical
Engineering, Computer Science and Informatics, 61-64.

[49] Yang, C., Novaliendry, D., Chen, J., Wattimena, F. Y., Renyaan, A. S., Lizar, Y., ... Nasution, T. (2020). Prediction of Mortalityinthe Hemodialysis Patient with Diabetes using Support Vector Machine. Revista Argentina de Clínica Psicológica, XXIX, 219-232.https://doi.org/10.24205/0327 6716.2020 .823

[50] Feladi, V., Hendriyani, Y., Dewi, I. P., Darni, R., \& Verawadina, U. (2020). The Profile of Technological Pedagogical and Content Knowledge of Information and Communication Technology Teachers. Test Engineering \& Management, 83, 1666-1673

[51] Bandri, S., Rukun, K., Sukardi., Verawardina, U., \& Ramadhani, D. (2020). The Validity of the Model of Employability Skills Required for Graduates to Enter the Workplace. Test Engineering \& Management, 83, 16381642

[52] Hendriyani, Y., Ramadhani, D., Nasution, T., Susanti, W., \& Verawardina, U. (2020). Examining Career Development of Informatics Engineering Vocational Education Students in the Industrial Revolution 4.0. International Journal of Innovation, Creativity and Change, 11(4), 275-298.

[53] Verawardina, U., Ramadhani, D., Susanti, W., Lubis, A. L., \& Simeru, A. (2020). Studying technology-based XXI Century Learning using MOOC in Education. International Journal of Psychosocial Rehabilitation, 24(9), 2644-2650

[54] Vitriani, Ali, G., Nanda, D. W., Syahril, Desnelita, Y., Satria, R., \& Verawadina, U. (2020). The validity of training models based on knowledge management systems. International Journal of Innovation, Creativity, and Change, 12(12), 726-741.

[55] Nofrianto, H., Jama, J., \& Indra, A. (2020). Validity of Cooperative-Discovery Learning Model to Improve Competencies of Engineering Students. Systematic Reviews in Pharmacy, 11(12), 1134-1138. 